\title{
COMT polymorphism regulates the hippocampal subfield volumes in first-episode, drug-naive patients with major depressive disorder
}

This article was published in the following Dove Press journal: Neuropsychiatric Disease and Treatment

\author{
Yuka Otsuka' \\ Shingo Kakeda ${ }^{2}$ \\ Koichiro Sugimoto ${ }^{2}$ \\ Asuka Katsuki' \\ Le Hoa Nguyen' \\ Ryohei Igatal \\ Keita Watanabe ${ }^{2}$ \\ Issei Ueda ${ }^{2}$ \\ Taro Kishi ${ }^{3}$ \\ Nakao Iwata ${ }^{3}$ \\ Yukunori Korogi $^{2}$ \\ Reiji Yoshimura' \\ 'Department of Psychiatry, University of \\ Occupational and Environmental Health, \\ Kitakyushu, Japan; ${ }^{2}$ Department of \\ Radiology, University of Occupational and \\ Environmental Health, Kitakyushu, Japan; \\ ${ }^{3}$ Department of Psychiatry, Fujita Health \\ University School of Medicine, Toyoake, \\ Japan
}

Purpose: Compared with healthy subjects (HS), patients with major depressive disorder (MDD) exhibit volume differences that affect the volume changes in several areas such as the limbic, cortical, subcortical, and white matter. Catechol-O-methyltransferase (COMT) is a methylation enzyme that catalyzes endogenous catecholamines. The Val158Met polymorphism of COMT has been reported to affect the dopamine (DA) levels, which plays an important role in psychiatric diseases. However, the relationships among both DA levels, COMT genotype, and brain morphology are complicated and controversial. In previous studies that investigated the hippocampal subfields, the greatest brain abnormalities in MDD patients were observed in Cornu Ammonis (CA)1 and the subiculum, followed by that in CA2-3. We have prospectively demonstrated the relationship between the singlenucleotide polymorphism of the Val158Met COMT gene (rs4680) and the hippocampal subfields in drug-naive MDD patients.

Patients and methods: In this study, we compared 27 MDD patients and 42 HS who were divided into groups based on their COMT genotype. The effects of the diagnosis, genotype, and genotype-diagnosis interaction related to CA1 and the subiculum volumes, as well as the whole-brain cortical thickness, were evaluated by performing a FreeSurfer statistical analysis of high-resolution magnetic resonance imaging (MRI) findings.

Results: The results revealed that there was a statistically significant interaction between the effects of diagnosis and genotype on the right subiculum (a component of the hippocampus). Conclusion: This Val158Met COMT polymorphism may influence the subiculum volume in drug-naive, first-episode MDD patients.

Keywords: major depressive disorder, COMT gene, brain morphology, hippocampal subfields volume, subiculum, surface-based morphometry

\section{Introduction}

Catechol-O-methyltransferase (COMT) on chromosome $22 \mathrm{q}^{1} 1^{1}$ is a methylation enzyme that catalyzes endogenous catecholamines via O-methylation in the presence of S-adenosylmethionine and magnesium ions. ${ }^{2}$ The dopamine (DA) levels are sensitive to the COMT levels; therefore, variations in COMT activity may be involved in the pathogenesis of various psychiatric and neurological diseases. ${ }^{3}$ COMT is widely expressed in the hippocampus and prefrontal cortex (PFC) ${ }^{4}$ and is involved in basic neuronal functions, such as synaptic plasticity. ${ }^{5}$

A functional missense mutation of chromosome 22q11 causes a single G-to-A base-pair substitution, resulting in a single-nucleotide polymorphism (SNP) in exon
Correspondence: Yuka Otsuka

Department of Psychiatry, University of Occupational and Environmental Health, I-I Iseigaoka, Yahatanishi-ku, Kitakyushu, Fukuoka 8078555, Japan

Tel +8 1936917253

Fax +81936924894

Email yukaharada08I0@med.uoeh-u.ac.jp 
4. This morphism changes codons $108 / 158$ from valine (Val) to methionine (Met), ${ }^{6}$ which in turn impairs the thermostability of mature proteins, ${ }^{7}$ thereby altering the DA levels in several brain regions ${ }^{8,9}$ and affects tasks of executive function, memory, and attention. ${ }^{10}$ The relationship between DA levels and brain function or morphology is complicated. The predictability of poor performance on the cognitive tasks by both deficient and excessive amounts of DA is best illustrated as an inverted U-shaped dose-response curve. ${ }^{11,12}$ An association between genetic factors and brain morphology seems to be based on the DA's ability. However, whether only the COMT polymorphism affects the brain abnormalities remains controversial. The brain abnormalities were recorded from both genetic factors and psychiatric diseases that regulate brain development or neurodegeneration, ${ }^{13}$ and not only gene polymorphism.

Structural neuroimaging findings have revealed brain volume reductions in multiple fronto-striatal-limbic regions of depressed patients, including the anterior cingulate cortex, the PFCs, the striatum, the hippocampus, and the amygdala. ${ }^{14-16}$ Recently, the hippocampus, a component of limbic structures, has been reported to be nonuniform in structure and comprises 26 subfields (13 left and 13 right) with distinct morphologies. ${ }^{17,18}$ These areas performed different functions in the memory system, ${ }^{19-22}$ and the subiculum and Cornu Ammonis (CA)1 plays a role in disambiguation during the working memory processes, ${ }^{23}$ CA1 and the subiculum, including its anterior region associated with emotional functioning, ${ }^{24}$ may play an important role in the pathophysiology of major depressive disorder (MDD). Owing to the fact that the subiculum plays a role in the hippocampal output from $\mathrm{CA} 1$ and $\mathrm{CA} 3,{ }^{25}$ a secondary effect was hypothesized to occur in the subiculum that is mediated by CA1 and CA3 damage owing to the high cortisol levels. ${ }^{26,27}$ Furthermore, female monkeys with depressive-like behaviors form a nonhuman primate model and have been reported to display smaller anterior CA1 and subiculum volumes than normal monkeys, although the entire hippocampal volume did not differ. ${ }^{28}$ The differences in the PFC and temporal lobe areas are not large enough as those in the subiculum in individuals with schizophrenia and mood disorders, ${ }^{29}$ suggesting that the abnormality may be localized to a hippocampal subfield.

To date, no studies have examined the comprehensive association between the COMT Val158Met polymorphism and hippocampal subfields. Therefore, we hypothesized that the impact of COMTVal158Met genotype may be localized in the CA1 and the subiculum, as suggested by previous studies, regarding the pathophysiology of MDD. The aim of this neuroimaging study was to comprehensively investigate the relationship between COMTVal158Met polymorphism and morphometric brain abnormalities in drug-naive, first-episode MDD patients, allowing observations without the effects of antidepressants.

\section{Materials and methods}

\section{Participants}

This study was performed in accordance with the Declaration of Helsinki, and the protocol used in this prospective study was approved by the Institutional Review Board of University of Occupational and Environmental Health in Kitakyushu, Japan. All the participants provided written informed consent to participate in the study. Most of the subjects who participated in this study also participated in an earlier published study ${ }^{30}$ that examined the relationship between cortical thickness and the serum cortisol levels in MDD by performing a surfacebased morphometry analysis using the FreeSurfer software program (http://surfer.nmr.mgh.harvard.edu).

Twenty-nine right-handed, treatment-naive patients experiencing their first episode of MDD were recruited. A psychiatrist (R.Y. with 28 years of experience in psychiatry) diagnosed the patients experiencing major depressive episodes using the Structured Clinical Interview according to the Diagnostic and Statistical Manual of Mental Disorders (DSM)-IV-TR. ${ }^{31}$ The 17-item Hamilton Rating Scale for Depression (HAMD17) was used to evaluate the severity of depression. ${ }^{32}$ Patients with a HAMD17 score $\leq 7$ were not considered eligible for the study. The exclusion criteria included any history of neurological diseases or other physical diseases and the presence of other disorders (ie, the evidence of schizoaffective disorder, bipolar disorder, Axis II personality disorder, or mental retardation). Forty-seven healthy subjects (HS) were also recruited via an interview conducted by the same psychiatrist using the Structured Clinical Interview for the DSM-IV, ${ }^{33}$ nonpatient edition. None of the participants had a history of any neuropsychiatric disorders or personality disorders. Of the 29 MDD patients, two were excluded because of change in diagnosis, and five of the $47 \mathrm{HS}$ were excluded to match with the patients in terms of age and sex. 27 MDD patients and 42 HS were divided into groups based on their COMT genotype as follows: 13 MDD patients with the Val/Val genotype (Val/Val-carriers), 14 MDD patients with either 
Met genotype (Val/Met $n=14$, or Met/Met $n=0$ ) (Metcarriers), $22 \mathrm{HS} \mathrm{Val} / \mathrm{Val}$, and $20 \mathrm{HS}$ with either Met genotype (Val/Met n=17, Met/Met n=3) (Table 1).

\section{Genotyping}

Each of the 69 participants evaluated in the neuroimaging study provided a blood sample from which DNA was extracted, and the COMT DNA was amplified using standard laboratory protocols. DNA was isolated from the peripheral blood mononuclear cells using the QIAamp DNA Mini-Kit (QIAGEN, Tokyo, Japan). The human COMT gene was amplified from the genomic DNA by performing PCR. The PCR products were purified enzymatically. The Big Dye Terminator v3.1 Cycle Sequencing Kit (Life Technologies Corporation, Waltham, MA, USA) was used to perform the sequencing reactions. The sequencing primers used were the same as the PCR primers. The sequences were read using the Applied Biosystem 3730xl DNA Analyzer (Life Technologies Corporation). Then, the sequencing output data were compared with a reference sequence (NC-000007.13). Each participant was homozygous for the Val allele (Val/Val genotype), heterozygous (Val/Met genotype), or homozygous for the Met allele (Met/Met genotype).

\section{Magnetic resonance imaging (MRI) acquisition}

The MRI data were obtained using the 3T MR System (Signa EXCITE 3T; GE Healthcare, Wankesha, WI, USA) with an 8-channel brain phased-array coil. The original T1 images were acquired using a 3D fast-spoiled gradient recalled acquisition with a steady state. The acquisition parameters were as follows: repetition time in ms/echo time in $\mathrm{ms} /$ inversion time in $\mathrm{ms}=10 / 4.1 / 700$; flip angle $=10$; field-of view $=24 \mathrm{~cm}$; section thickness $=1.2 \mathrm{~mm}$, and resolution $=0.9 \times 0.9 \times 1.2 \mathrm{~mm}$. All the images were corrected for image distortion owing to gradient nonlinearity using the Grad Warp software program ${ }^{34}$ and intensity inhomogeneity using the "N3" function. ${ }^{35}$

\section{Image processing}

Whole-brain analyses using surfaced-based morphometry (SBM)

The regional cortical thickness was estimated using the FreeSurfer software version 6.0 (http://www.freesurfer.net/ fswiki/FreeSurferWiki), which has been well documented and is freely available online. The technical details of the cortical thickness analysis have been previously described elsewhere. $^{36}$ The entire cortex of each participant was visually inspected, and the topological defects were manually corrected. The cortical thickness measurements were performed by reconstructing representations of the gray matter-white matter boundary ${ }^{36,37}$ and the pial surface. Then, the distance between these surfaces at each point across the cortical mantle was calculated. For each participant, the regional thickness value at each vertex was mapped onto the surface of an average brain template. This procedure enabled the visualization of the data across the entire cortical surface. The data were resampled for all the participants onto a common spherical coordinate system. $^{37}$ The cortical map of each participant was smoothed using a 10-mm kernel in full width at halfmaximum (FWHM) for the cortical analyses.

\section{Volumetry of hippocampal subfields}

Because the SBM analysis could not analyze the hippocampus, we performed a hippocampal subfield analysis using the automated hippocampal subfield segmentation method. The FreeSurfer software version 6.0 was used to calculate the hippocampal subfield volumes (www.freesur fer.net/fswiki/HippocampalSubfields). ${ }^{38}$ The calculated subregions included the CA1, CA3, and CA4; granule cell layer of the dentate gyrus (GC-DG); fimbria;

Table I Demographic and clinical characteristics among 4 groups

\begin{tabular}{|l|l|l|l|l|l|}
\hline & \multicolumn{2}{l}{ MDD patients } & Healthy subjects & $p$-value \\
\hline & Val/Val $(\mathbf{n}=13)$ & Met-carrier $(\mathbf{n}=14)$ & Val/Val $(\mathbf{n}=22)$ & Met-carrier $(\mathbf{n}=20)$ & \\
\hline Male/female & $8 / 5$ & $8 / 6$ & $16 / 6$ & $15 / 5$ & $p=0.657$ \\
\hline Age (years) & $41.92 \pm 11.06$ & $49.29 \pm 13.18$ & $41.45 \pm 12.79$ & $40.1 \pm 10.74$ & $p=0.153$ \\
\hline HAMDI7 & $21.08 \pm 7.1$ & $21.57 \pm 5.26$ & & $p=0.884$ \\
\hline HWE & $p=0.0690$ & $p=0.908$ & & \\
\hline
\end{tabular}

Abbreviations: MDD, major depressive disorder; HAMDI7, I7-item Hamilton Rating Scale for Depression; HWE, Hardy-Weinberg equilibrium; Met, methionine; Val, valine. 


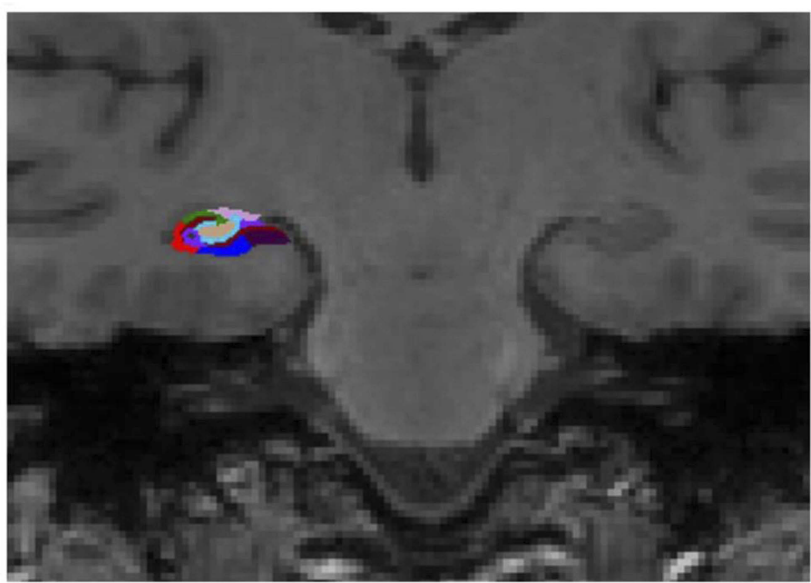

Figure I Representative subdivision of the hippocampal subfields. The mask of each region was overlapped on the coronal TI-weighted images. Color classification: parasubiculum = yellow; presubiculum = black; subiculum = blue; Cornu Ammonis (CA) I = red; CA3 = dark green; CA4 = brown; granule cell layer of dentate gyrus (GC-DG) = sky blue; hippocampus-amygdala-transition-area (HATA) = green; fimbria $=$ purple; molecular layer hippocampus $(\mathrm{HP})=$ dark brown; hippocampal fissure $=$ dark purple; hippocampal tail $=$ gray .

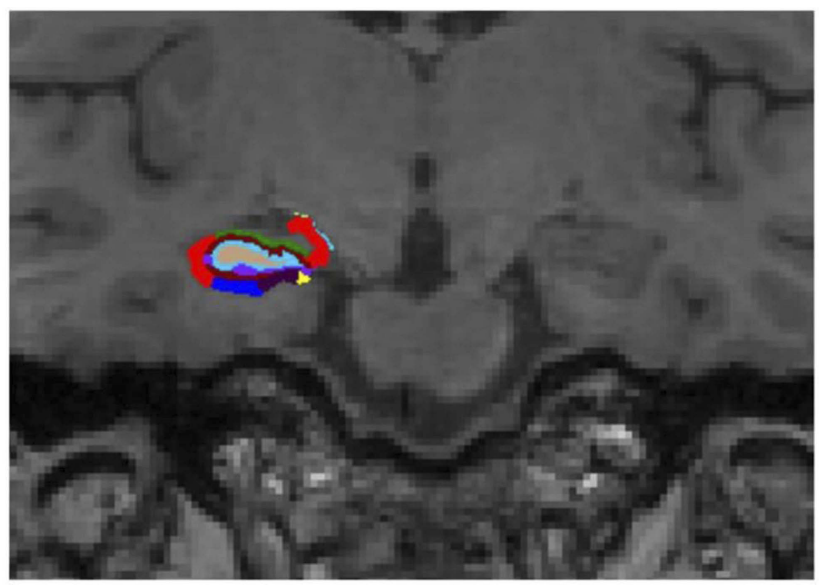

Figure 2 Representative subdivision of the hippocampal subfields. The mask of each region was overlapped on the coronal TI-weighted images. Color classification: parasubiculum = yellow; presubiculum = black; subiculum $=$ blue; Cornu Ammonis $(C A) I=$ red; $C A 3$ = dark green; $C A 4$ = brown; granule cell layer of dentate gyrus (GC-DG) = sky blue; hippocampus-amygdala-transition-area (HATA) = green; fimbria $=$ purple; molecular layer hippocampus $(\mathrm{HP})=$ dark brown; hippocampal fissure $=$ dark purple; hippocampal tail $=$ gray .

subiculum; presubiculum; parasubiculum; hippocampal fissure; molecular layer; hippocampus-amygdalatransition-area (HATA); hippocampal tail; and whole hippocampus (Figures 1-3). We calculated the volumes of CA1 and subiculum. The technical details of these procedures have been described previously. ${ }^{39}$

\section{Statistical analysis}

To analyze the demographic and clinical characteristics of the patients, an ANOVA was performed to test for

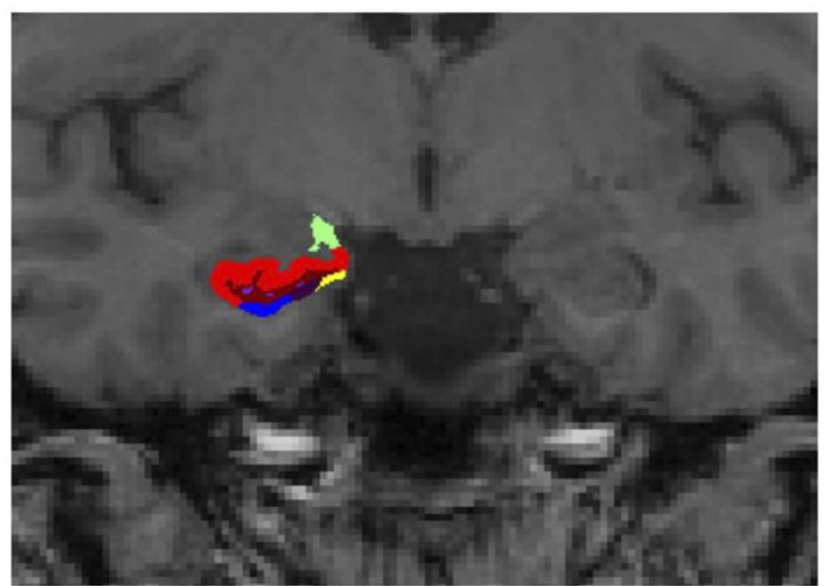

Figure 3 Representative subdivision of the hippocampal subfields. The mask of each region was overlapped on the coronal TI-weighted images. Color classification: parasubiculum = yellow; presubiculum = black; subiculum = blue; Cornu Ammonis $(C A) I=$ red; $C A 3$ = dark green; $C A 4$ = brown; granule cell layer of dentate gyrus (GC-DG) = sky blue; hippocampus-amygdala-transition-area (HATA) = green; fimbria $=$ purple; molecular layer hippocampus $(\mathrm{HP})=$ dark brown; hippocampal fissure $=$ dark purple; hippocampal tail $=$ gray.

differences in the age among the HS with $\mathrm{Val} / \mathrm{Val}$, HS with either Met, MDD patients with Val/Val, and MDD patients with either Met. Fisher's exact test was performed to evaluate the differences in sex among the groups. Mann-Whitney $U$ test was performed to compare the total HAMD17 scores between the MDD patients with $\mathrm{Val} / \mathrm{Val}$ and those with either Met.

A voxel-wise statistical analysis was performed using a surface-based analysis with the FreeSurfer statistical tool QDEC after performing a 10-mm FWHM kernel smoothing. Then, a general linear model was applied at each vertex. The morphological changes in the cortical thickness were assessed by establishing a full factorial model with the diagnosis and genotype status as independent variables. Age and sex were included as covariates of no interest in all analyses to control for confounding variables. The following $t$-test comparisons for $2 \times 2$ factorial designs were performed: (a) diagnosis effects (MDD versus HS), (b) genotype effect, and (c) genotype-diagnosis interaction. In addition, age and sex were included as "nuisance factors" to control for the confounding variables. Because the controls subjects and MDD patients showed different cortical evolution rates, different offsets and different slopes were employed in this study. To correct for multiple comparisons, a Monte-Carlo simulation was performed for the cluster analysis. The cluster-forming threshold was set at $p<0.05$. Then, the clusters were tested against an empirical null distribution of a maximum cluster size generated using synthesized Z-distributed data across 
10,000 permutations to produce cluster-wise $p$-values that were fully corrected for multiple comparisons.

We used two-way ANOVA to evaluate the effects of the diagnosis, genotype, and genotype-diagnosis interaction on CA1 and subiculum volumes, followed by Bonferroni test for correcting multiple comparisons. The individual hippocampal subfield volumes were normalized for intersubject variation for head size by dividing the hippocampal subfield volumes by the total intracranial volume. Moreover, because only the genotype-diagnosis interaction was identified using two-way ANOVA, we compared each pair of combinations divided based on genotype and diagnosis, and we confirmed simple main effects.

The statistical analyses were considered significant at $p<0.05$. All statistical analyses were performed using the EZR software version 1.35 (Saitama Medical Center, Jichi Medical University, Saitama, Japan), ${ }^{40}$ which is a graphical user interface in $\mathrm{R}$ (The $\mathrm{R}$ Foundation for Statistical Computing, Vienna, Austria). Specifically, EZR is a modified version of $\mathrm{R}$ commander with additional statistical functions that is frequently used in biostatistics.

\section{Results}

\section{Demographic and clinical data}

Table 1 shows the demographic and clinical data of the enrolled subjects. In this sample, the distributions of the genotypes in the HS and MDD patients were in agreement with the Hardy-Weinberg equilibrium (Table 1). No significant differences were observed among the 4 groups (HS with Val/Val, HS with either Met, MDD with Val/ Val, and MDD with either Met) in the age and sex distributions between the HS and MDD patients. Analyses showed no significant interaction between genotype and sex nor between genotype and age terms, implying that genotype effects were stable across the age range. No significant differences were observed in the HAMD17 scores between the MDD patients with Val/Val and those with either Met.

\section{Whole-brain analysis using SBM}

In any brain area, no significant differences were noted in the cortical thickness between the $42 \mathrm{HS}$ and $27 \mathrm{MDD}$ patients, that is, no diagnosis effects were observed (HS with $\mathrm{Val} / \mathrm{Val}$ and Met carriers versus MDD with $\mathrm{Val} / \mathrm{Val}$ and Met carriers), and no COMT genotype effects were observed (MDD and HS with Val/Val versus MDD and HS with Met carriers). Regarding the COMT genotype-diagnosis interaction (diagnosis effects in Val/Val individuals versus diagnosis effects in Met-carrier individuals), we did not identify any brain area wherein a genotype effect differed significantly between the diagnosis groups $(p<0.05$, FWE corrected).

\section{Volumetry of hippocampal subfields}

In two-way ANOVA, there was a statistically significant interaction between the effects of diagnosis and genotype on the right subiculum $(p<0.05)$ (Table 2) (Figure 4). Bonferroni adjustment $p$-value for multiple comparisons of interaction was 0.042 . The right subiculum volume affected both diagnosis and genotype; however, the directionality of effects was different. Val/Val individuals more decreased the volume than Met carriers when they developed MDD. Moreover, Tukey's HSD post hoc tests were carried out to confirm simple main effects. However, we did not observe any simple main effects of diagnosis and genotype associated with the right subiculum volume (Table 3 ).

\section{Discussion}

To the best of our knowledge, this study provides the first evidence of a relationship between the hippocampal subfield

Table 2 The diagnosis effect, genotype effect, and genotype-diagnosis interaction on CAI and subiculum

\begin{tabular}{|c|c|c|c|c|c|c|c|}
\hline \multicolumn{2}{|c|}{ Hippocampal subfields } & \multicolumn{2}{|c|}{ Disgnosis effect } & \multicolumn{2}{|c|}{ Genotype effect } & \multicolumn{2}{|c|}{ Genotype-diagnosis interaction } \\
\hline & & F-value & Adjusted $p$-value & F-value & Adjusted $p$-value & F-value & Adjusted $p$-value \\
\hline \multirow[t]{2}{*}{ Left } & CAI & 0.0058 & 3.76 & 0.0882 & 3.07 & 0.853 & $\mathrm{I} .44$ \\
\hline & Subiculum & 0.977 & 1.31 & 0.0982 & 3.02 & 3.334 & 0.29 \\
\hline \multirow[t]{2}{*}{ Right } & CAI & 0.113 & 2.95 & 0.136 & 2.85 & 3.634 & 0.24 \\
\hline & Subiculum & 0.515 & 1.904 & 0.105 & 2.99 & 6.926 & $0.042 *$ \\
\hline
\end{tabular}

Note: *Significant genotype-diagnosis interactions $(p<0.05)$. Adjusted $p$-value: adjusted $p$-value by Bonferroni method. Abbreviation: CA, Cornu Ammonis. 
The right subiculum volume

dividing by the total intracranial volume

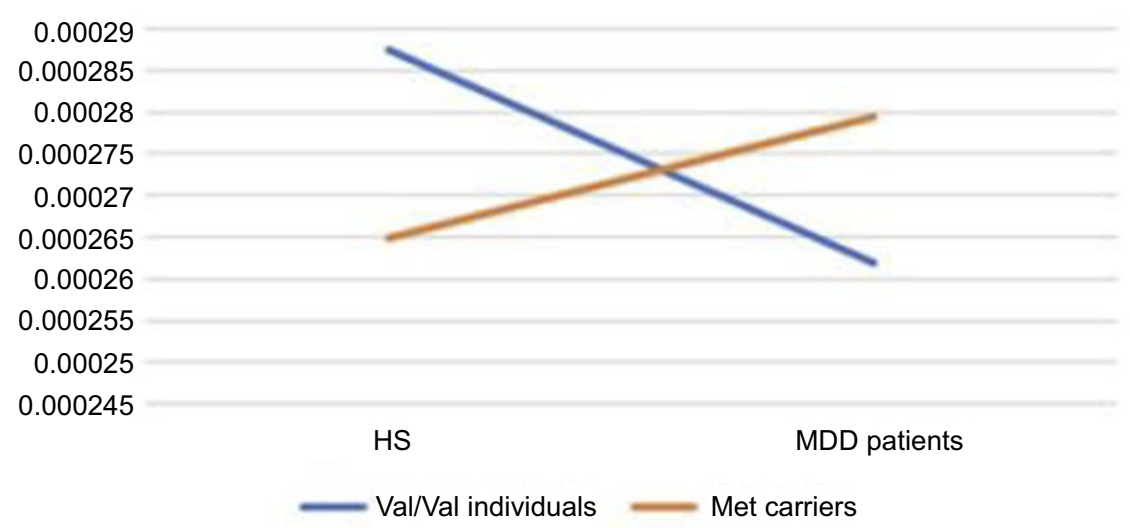

Figure 4 The visualization of the association between diagnosis and genotype in right subiculum volume. Abbreviations: HS, healthy subjects; MDD, major depressive disorder.

Table 3 The comparison of each pair of combinations divided based on genotype-diagnosis groups in the right subiculum

\begin{tabular}{|l|l|l|l|l|l|}
\hline & $\begin{array}{l}\text { Groups being } \\
\text { campared }\end{array}$ & $\begin{array}{l}\text { Difference in } \\
\text { means }\end{array}$ & Lower $\mathbf{C l}$ & $\begin{array}{l}\text { Upper } \\
\text { Cl }\end{array}$ & $\begin{array}{l}\text { Adjusted } \\
\boldsymbol{p} \text {-value }\end{array}$ \\
\cline { 3 - 6 } & & difference & lower & upper & $\boldsymbol{p}$ adjusted \\
\hline Comparisons with MDD with Val/ & MDD with Met carriers & $1.77 \times 10^{-5}$ & $-1.38 \times 10^{-5}$ & $4.92 \times 10^{-5}$ & $p=0.456$ \\
Val & HS with Val/Val & $2.56 \times 10^{-5}$ & $-5.42 \times 10^{-5}$ & $2.96 \times 10^{-6}$ & $p=0.0945$ \\
Comparisons with MDD & HS with Met carriers & $3.01 \times 10^{-6}$ & $-2.61 \times 10^{-5}$ & $3.21 \times 10^{-5}$ & $p=0.993$ \\
with Met carriers & HS with Val/Val & $7.98 \times 10^{-6}$ & $-3.59 \times 10^{-5}$ & $2.00 \times 10^{-5}$ & $p=0.875$ \\
Comparisons with HS with Val/Val & HS with Met carriers & $1.47 \times 10^{-5}$ & $-1.38 \times 10^{-5}$ & $4.32 \times 10^{-5}$ & $p=0.531$ \\
& HS with Met carriers & $2.26 \times 10^{-5}$ & $-4.79 \times 10^{-5}$ & $2.63 \times 10^{-6}$ & $p=0.0948$ \\
\hline
\end{tabular}

Abbreviations: $\mathrm{Cl}$, confidence interval; $\mathrm{HS}$, healthy subjects; MDD, major depressive disorder.

volumes and COMT in first-episode, drug-naive MDD patients. In this study, there was a statistically significant interaction between the effects of diagnosis and COMT Val158Met polymorphism on the right subiculum of the hippocampus despite the lack of change in the whole-brain cortical thickness. Thus, the brain changes that are likely associated with COMT Val158Met polymorphisms in MDD patients occur in localized hippocampal subfields. Our results also support the hypotheses that the subiculum may be involved in the pathophysiology of MDD and that the hippocampal subfields abnormality does not always appear in parallel with whole-brain abnormality.

In this study, no main effect of the genotype was observed in the brain abnormalities. However, whether the COMT genotype directly affects the brain volume remains controversial. Previous studies among healthy volunteer have demonstrated such diverse results. Some researchers have reported that carriers of Met alleles within the Val158Met COMT polymorphism have exhibited increased volume/cortical thickness in the frontal and temporal cortices ${ }^{41,42}$ and posterior cingulate cortex. ${ }^{43}$ Cerasa et al reported similar notion about the existence of this relationship; however, Honea et al found that Val alleles and not Met alleles were associated with increased volume in the frontal cortex and that Met alleles affected greater tissue volume of the hippocampus. ${ }^{44,45}$ In contrast, no data revealed statistical association between gray matter volume and COMT genotype in whole-brain analysis, ${ }^{46,47}$ and no such association has been found in the frontal cortex $^{48}$ or hippocampal volume ${ }^{49}$ and COMT genotype in studies performing the region of interest analyses. Thus, the relationship between the COMT genotype and brain morphology remains controversial. The discrepancies among these previous reports ${ }^{41-45,49}$ may be due to the differences in the image analysis methods and study design. 
Regarding the main effect of the diagnosis, the volumes of all hippocampal subfields did not significantly differ between the MDD and HS. The hippocampus is believed to play an important role in MDD. For instance, the hippocampus is involved in verbal memory functions and other complex behaviors, including stress responses, emotions, sensory-motor integrations, and goal-directed activity, all of which may be disrupted in mood disorder. Previous studies have reported that the volume of the hippocampus is reduced in both MDD and anxiety disorders, ${ }^{50-53}$ and a reduction in the hippocampal volume has been related to the severity and duration of depression disorder. $^{54,55}$

Among the hippocampal subfields, the subiculum is located in the inferomedial area of the hippocampus and, thus, serves as a connection between the CA1 and the entorhinal cortex. The CA1 and the subiculum, which belong to the anterior region, play an important role in emotional functioning ${ }^{24}$ and may be associated with the pathophysiology of MDD. Our results did not support those of previous studies because MDD patients exposed to chronic stress exhibit structural alternations in the hippocampal and PFC regions; ${ }^{56-59}$ therefore, the brain abnormalities in MDD might be due to both genetic and epigenetic factors, although we did not involve the epigenetic factors.

Based on the above-mentioned results, there is neither the main effect of diagnosis nor the main effect of genotype, but we found a statistically significant interaction only between the effects of diagnosis and COMT Val158Met polymorphism on the right subiculum of the hippocampus. The directionality of effects to subiculum volume was different from diagnosis and genotype. The volume reduction observed in certain regions of the hippocampus in MDD patients could be explained by the variation in COMT gene, which partially affects the pathophysiology on MDD. Certain other genes, hormones, neurotrophic factors, and cytokines are likely to affect the hippocampal volume in MDD patients.

This study has several limitations, and the results of the study are preliminary. First, the sample size was small as patients from only one institution were recruited, and the sample was heterogeneous because the HS group consisted of staff from our institution, their relatives (by marriage), and close friends. Biologically related relatives were excluded. Second, we combined Val/Met and Met/Met as Met carriers because only a few subjects were Met/Met homozygous. In the rs4680 SNP, the frequency of Met- allele Asian individuals was low, which is a common occurrence in the study of a particular polymorphism (global: $\mathrm{G}=0.53748, \quad \mathrm{~A}=0.46252 ; \quad$ Asian: $\mathrm{G}=0.6199$, $\mathrm{A}=0.3801$ ). Thus, we could not evaluate the differences in the subiculum volume between the Val/Met and Met/ Met-carriers. Further studies including epigenetic factors, such as present stressors involving larger and homogeneous samples, are expected to produce robust results regarding volume reduction in the subfields of the hippocampus and the COMT Val158/Met genotype in firstepisode, drug-naive MDD patients.

In conclusion, the COMT Val158Met polymorphism was associated with differences in several areas of the hippocampus in first-episode, drug-naive MDD patients. The different results for the diagnosis effect, genotype effects, and genotype-diagnosis interaction by each hippocampal subfield observed in the present study may play an important role in the pathogenesis of MDD.

\section{Acknowledgments}

This study was supported by a Health and Labor Research Grant in Japan (\#1401010101).

\section{Disclosure}

The authors report no conflicts of interest in this work.

\section{References}

1. Dickinson D, Elvevag B. Genes, cognition and brain through a COMT lens. Neuroscience. 2009;164(1):72-87. doi:10.1016/j. neuroscience.2009.05.014

2. Lotta T, Vidgren J, Tilgmann C, et al. Kinetics of human soluble and membrane-bound catechol O-methyltransferase: a revised mechanism and description of the thermolabile variant of the enzyme. Biochemistry. 1995;34(13):4202-4210.

3. Bertocci B, Miggiano V, Da Prada M, Dembic Z, Lahm HW, Malherbe P. Human catechol-O-methyltransferase: cloning and expression of the membrane-associated form. Proc Natl Acad Sci U S A. 1991;88(4):1416-1420. doi:10.1073/pnas.88.4.1416

4. Matsumoto M, Weickert CS, Beltaifa S, et al. Catechol O-methyltransferase (COMT) mRNA expression in the dorsolateral prefrontal cortex of patients with schizophrenia. Neuropsychopharmacology. 2003;28(8):1521-1530. doi:10.1038/sj. npp. 1300218

5. Liu W, Ge T, Leng Y, et al. The role of neural plasticity in depression: from hippocampus to prefrontal cortex. Neural Plast. 2017;2017:6871089. doi:10.1155/2017/6971206

6. Lachman HM, Papolos DF, Saito T, Yu YM, Szumlanski CL, Weinshilboum RM. Human catechol-O-methyltransferase pharmacogenetics: description of a functional polymorphism and its potential application to neuropsychiatric disorders. Pharmacogenetics. 1996;6 (3):243-250.

7. Ira E, Zanoni M, Ruggeri M, Dazzan P, Tosato S. COMT, neuropsychological function and brain structure in schizophrenia: a systematic review and neurobiological interpretation. $J$ Psychiatry Neurosci. 2013;38(6):366-380. doi:10.1503/jpn.120178 
8. Bilder RM, Volavka J, Czobor P, et al. Neurocognitive correlates of the COMT Val(158)Met polymorphism in chronic schizophrenia. Biol Psychiatry. 2002;52(7):701-707.

9. Tosato S, Bonetto C, Di Forti M, et al. Effect of COMT genotype on aggressive behaviour in a community cohort of schizophrenic patients. Neurosci Lett. 2011;495(1):17-21. doi:10.1016/j. neulet.2011.03.018

10. Bilder RM, Volavka J, Lachman HM, Grace AA. The catechol-O-methyltransferase polymorphism: relations to the tonic-phasic dopamine hypothesis and neuropsychiatric phenotypes. Neuropsychopharmacol. 2004;29(11):1943-1961. doi:10.1038/sj. npp. 1300542

11. Williams GV, Goldman-Rakic PS. Modulation of memory fields by dopamine D1 receptors in prefrontal cortex. Nature. 1995;376 (6541):572-575. doi:10.1038/376572a0

12. Vijayraghavan $\mathrm{S}$, Wang $\mathrm{M}$, Birnbaum $\mathrm{SG}$, Williams $\mathrm{GV}$, Arnsten AFT. Inverted-U dopamine D1 receptor actions on prefrontal neurons engaged in working memory. Nat Neurosci. 2007;10 (3):376-384. doi:10.1038/nn1846

13. Ide S, Kakeda S, Watanabe K, et al. Relationship between a BDNF gene polymorphism and the brain volume in treatment-naive patients with major depressive disorder: a VBM analysis of brain MRI. Psychiatry Res. 2015;233(2):120-124. doi:10.1016/j. pscychresns.2015.05.016

14. Alexopoulos GS. Frontostriatal and limbic dysfunction in late-life depression. Am J Geriatr Psychiatry. 2002;10(6):687-695.

15. Sexton CE, Mackay CE, Ebmeier KP. A systematic review and meta-analysis of magnetic resonance imaging studies in late-life depression. Am J Geriatr Psychiatry. 2013;21(2):184-195. doi:10.1016/j.jagp.2012.10.019

16. Naismith SL, Norrie LM, Mowszowski L, Hickie IB. The neurobiology of depression in later-life: clinical, neuropsychological, neuroimaging and pathophysiological features. Prog Neurobiol. 2012;98 (1):99-143. doi:10.1016/j.pneurobio.2012.05.009

17. Small SA, Schobel SA, Buxton RB, Witter MP, Barnes CA. A pathophysiological framework of hippocampal dysfunction in ageing and disease. Nat Rev Neurosci. 2011;12(10):585-601. doi: $10.1038 / \mathrm{nrn} 3085$

18. Hibar DP, Adams HHH, Jahanshad N, et al. Novel genetic loci associated with hippocampal volume. Nat Commun. 2017;8:13624. doi:10.1038/ncomms 13624

19. Squire LR. Memory systems of the brain: a brief history and current perspective. Neurobiol Learn Mem. 2004;82(3):171-177. doi:10.1016/j.nlm.2004.06.005

20. Moscovitch M, Nadel L, Winocur G, Gilboa A, Rosenbaum RS. The cognitive neuroscience of remote episodic, semantic and spatial memory. Curr Opin Neurobiol. 2006;16(2):179-190. doi:10.1016/j. conb.2006.03.013

21. Bakker A, Kirwan CB, Miller M, Stark CE. Pattern separation in the human hippocampal CA3 and dentate gyrus. Science. 2008;319 (5870):1640-1642. doi:10.1126/science.1152882

22. Wolk DA, Dunfee KL, Dickerson BC, Aizenstein HJ, DeKosky ST. A medial temporal lobe division of labor: insights from memory in aging and early Alzheimer disease. Hippocampus. 2011;21 (5):461-466. doi:10.1002/hipo.20779

23. Newmark RE, Schon K, Ross RS, Stern CE. Contributions of the hippocampal subfields and entorhinal cortex to disambiguation during working memory. Hippocampus. 2013;23(6):467-475. doi:10.1002/hipo.22106

24. Moser MB, Moser EI. Functional differentiation in the hippocampus. Hippocampus. 1998;8(6):608-619. doi:10.1002/(SICI)1098-1063(1998)8:6<608::AID-HIPO3>3.0.CO;2-7

25. O’Mara SM, Commins S, Anderson M, Gigg J. The subiculum: a review of form, physiology and function. Prog Neurobiol. 2001;64(2):129-155.
26. Alfarez DN, De Simoni A, Velzing EH, et al. Corticosterone reduces dendritic complexity in developing hippocampal CA1 neurons. Hippocampus . 2009;19(9):828-836. doi:10.1002/hipo.20566.

27. Magarin os AM, McEwen BS. Stress-induced atrophy of apical dendrites of hippocampal CA3c neurons: comparison of stressors. Neuroscience. 1995;69(1):83-88.

28. Willard SL, Riddle DR, Forbes ME, Shively CA. Cell number and neuropil alterations in subregions of the anterior hippocampus in a female monkey model of depression. Biol Psychiatry. 2013;74 (12):890-897. doi:10.1016/j.biopsych.2013.03.013

29. Rosoklija G, Toomayan G, Ellis SP, et al. Structural abnormalities of subicular dendrites in subjects with schizophrenia and mood disorders: preliminary findings. Arch Gen Psychiatry. 2000;57 (4):349-356.

30. Liu X, Kakeda S, Watanabe K, et al. Relationship between the cortical thickness and serum cortisol levels in drug-naive, first-episode patients with major depressive disorder: a surface-based morphometric study. Depress Anxiety. 2015;32 (9):702-708. doi:10.1002/da.22401

31. Association. AP. Diagnostic and Statistical Manual of Mental Disorders. Vol. 4. Washington, DC: American Psychiatric Association; 2000.

32. Hamilton M. Development of a rating scale for primary depressive illness. Br J Soc Clin Psychol. 1967;6(4):278-296.

33. First MSR, Gibbon M, Williams J. Structured clinical interview for DSM-IV axis I disorders (SCID-I), clinician version. Administration Booklet. Washington, DC: American Psychiatric Publishing; 2012.

34. Jovicich J, Czanner S, Greve D, et al. Reliability in multi-site structural MRI studies: effects of gradient non-linearity correction on phantom and human data. NeuroImage. 2006;30(2):436-443. doi:10.1016/j.neuroimage.2005.09.046

35. Sled JG, Zijdenbos AP, Evans AC. A nonparametric method for automatic correction of intensity nonuniformity in MRI data. IEEE Trans Med Imaging. 1998;17(1):87-97. doi:10.1109/42.668698

36. Dale AM, Fischl B, Sereno MI. Cortical surface-based analysis. I. Segmentation and surface reconstruction. NeuroImage. 1999;9 (2):179-194. doi:10.1006/nimg.1998.0395

37. Fischl B, Dale AM. Measuring the thickness of the human cerebral cortex from magnetic resonance images. Proc Natl Acad Sci U S A. 2000;97(20):11050-11055. doi:10.1073/pnas.200033797

38. Van Leemput K, Bakkour A, Benner T, et al. Automated segmentation of hippocampal subfields from ultra-high resolution in vivo MRI. Hippocampus. 2009;19(6):549-557. doi:10.1002/hipo.20615

39. Iglesias JE, Augustinack JC, Nguyen K, et al. A computational atlas of the hippocampal formation using ex vivo, ultra-high resolution MRI: application to adaptive segmentation of in vivo MRI. NeuroImage. 2015;115:117-137. doi:10.1016/j.neuroimage.2015.04.042

40. Kanda Y. Investigation of the freely available easy-to-use software 'EZR' for medical statistics. Bone Marrow Transplant. 2013;48 (3):452-458. doi:10.1038/bmt.2012.244

41. Shaw P, Wallace GL, Addington A, Evans A, Rapoport J, Giedd JN. Effects of the Val158Met catechol-O-methyltransferase polymorphism on cortical structure in children and adolescents. Mol Psychiatry. 2009;14(4):348-349. doi:10.1038/mp.2008.121

42. Cerasa A, Cherubini A, Quattrone A, et al. Met158 variant of the catechol-O-methyltransferase genotype is associated with thicker cortex in adult brain. Neuroscience. 2010;167(3):809-814. doi:10.1016/j.neuroscience.2010.02.040

43. Tian T, Qin W, Liu B, et al. Catechol-O-methyltransferase Val158Met polymorphism modulates gray matter volume and functional connectivity of the default mode network. PLoS One. 2013;8(10):e78697. doi:10.1371/journal.pone.0078697

44. Honea R, Verchinski BA, Pezawas L, et al. Impact of interacting functional variants in COMT on regional gray matter volume in human brain. Neuroimage. 2009;45(1):44-51. doi:10.1016/j. neuroimage.2008.10.064 
45. Cerasa A, Gioia MC, Labate A, Liguori M, Lanza P, Quattrone A. Impact of catechol-O-methyltransferase Val(108/158) Met genotype on hippocampal and prefrontal gray matter volume. Neuroreport. 2008;19(4):405-408. doi:10.1097/WNR.0b013e3282f5f784

46. Barnes A, Isohanni M, Barnett JH, et al. No association of COMT (Val158Met) genotype with brain structure differences between men and women. PLoS One. 2012;7(3):e33964. doi:10.1371/journal.pone.0033964

47. Ohnishi T, Hashimoto R, Mori T, et al. The association between the Val158Met polymorphism of the catechol-O-methyl transferase gene and morphological abnormalities of the brain in chronic schizophrenia. Brain. 2006;129(Pt 2):399-410. doi:10.1093/brain/awh702

48. Ho BC, Wassink TH, O’Leary DS, Sheffield VC, Andreasen NC. Catechol-O-methyl transferase Val158Met gene polymorphism in schizophrenia: working memory, frontal lobe MRI morphology and frontal cerebral blood flow. Mol Psychiatry. 2005;10(3):229,287-298. doi:10.1038/sj.mp.4001652

49. Dutt A, McDonald C, Dempster E, et al. The effect of COMT, BDNF, 5-HTT, NRG1 and DTNBP1 genes on hippocampal and lateral ventricular volume in psychosis. Psychol Med. 2009;39(11):1783-1797. doi:10.1017/S0033291709990316

50. Campbell S, Marriott M, Nahmias C, MacQueen GM. Lower hippocampal volume in patients suffering from depression: a meta-analysis. Am J Psychiatry. 2004;161(4):598-607. doi:10.1176/appi.ajp.161.4.598

51. Jacobs RH, Jenkins LM, Gabriel LB, et al. Increased coupling of intrinsic networks in remitted depressed youth predicts rumination and cognitive control. PLoS One. 2014;9(8):e104366. doi:10.1371/ journal.pone. 0104366
52. Lamers F, Cui L, Hickie IB, et al. Familial aggregation and heritability of the melancholic and atypical subtypes of depression. J Affect Disord. 2016;204:241-246. doi:10.1016/j.jad.2016.06.040

53. Oathes DJ, Patenaude B, Schatzberg AF, Etkin A. Neurobiological signatures of anxiety and depression in resting-state functional magnetic resonance imaging. Biol Psychiatry. 2015;77(4):385-393. doi:10.1016/j.biopsych.2014.08.006

54. Bell-McGinty S, Butters MA, Meltzer CC, Greer PJ, Reynolds CF 3rd, Becker JT. Brain morphometric abnormalities in geriatric depression: long-term neurobiological effects of illness duration. Am J Psychiatry. 2002;159(8):1424-1427. doi:10.1176/appi.ajp.159.8.1424

55. MacQueen GM, Campbell S, McEwen BS, et al. Course of illness, hippocampal function, and hippocampal volume in major depression. Proc Natl Acad Sci U S A. 2003;100(3):1387-1392. doi:10.1073/ pnas.0337481100

56. Radley JJ, Rocher AB, Miller M, et al. Repeated stress induces dendritic spine loss in the rat medial prefrontal cortex. Cerebral Cortex. 2006;16(3):313-320.

57. Cook SC, Wellman CL. Chronic stress alters dendritic morphology in rat medial prefrontal cortex. $J$ Neurobiol. 2004;60(2):236-248. doi:10.1002/neu.20025

58. Cerqueira JJ, Pego JM, Taipa R, Bessa JM, Almeida OF, Sousa N. Morphological correlates of corticosteroid-induced changes in prefrontal cortex-dependent behaviors. $J$ Neurosci. 2005;25 (34):7792-7800. doi:10.1523/JNEUROSCI.1598-05.2005

59. McEwen BS. Stress and hippocampal plasticity. Annu Rev Neurosci. 1999;22:105-122. doi:10.1146/annurev.neuro.22.1.105
Neuropsychiatric Disease and Treatment

\section{Publish your work in this journal}

Neuropsychiatric Disease and Treatment is an international, peerreviewed journal of clinical therapeutics and pharmacology focusing on concise rapid reporting of clinical or pre-clinical studies on a range of neuropsychiatric and neurological disorders. This journal is indexed on PubMed Central, the 'PsycINFO' database and CAS, and is the official journal of The International Neuropsychiatric Association (INA). The manuscript management system is completely online and includes a very quick and fair peer-review system, which is all easy to use. Visit http://www.dovepress.com/testimonials.php to read real quotes from published authors. 